\title{
AN ELECTRONIC "ANGLE MARKER" FOR THE SIEMENS TEXTURE DIFFRACTOMETER
}

\author{
J. S. KALLEND \\ Illinois Institute of Technology \\ Chicago, Illinois 60616 USA
}

(Received June 16, 1980)

\begin{abstract}
An electronic circuit is described which produces output pulses in response to preselected increments in specimen rotation on a Siemens Texture Diffractometer. The output pulses give an accurate indication of the specimen position, and may be used to trigger $X$-ray scaler read-out and reset operations for automatic data logging. The accuracy with which specimen position is determined is unaffected by variations in line frequency of the goniometer motor electrical supply, or by goniometer motor inertia when it is first switched on.
\end{abstract}

\section{INTRODUCTION}

Throughout the world there are a large number of laboratories making use of the Siemens Texture Diffractometer for texture measurements. As designed, this instrument was intended to be used in conjunction with a rate meter whose output, indicating the intensity of detected $\mathrm{x}$-rays, was recorded on a strip chart for subsequent analysis. ${ }^{1}$ To assist in this analysis an "angle marker" switch was incorporated in the specimen mount, which produced an output pulse for every $360^{\circ}$ of specimen azimuth rotation, and which was recorded on the same chart as the $\mathrm{X}$-ray intensities.

In recent years, $\mathrm{X}$-ray laboratories have been making increasing use of automatic digital data recording, and subsequent computer analysis, in which the $x$-ray count is accumulated in a scaler for a short time period and then transmitted to a storage device of some kind. In the most usual set up, the total elapsed time from the start of an experiment is used to determine the specimen position. However, a serious problem may arise if this method is used with the Siemens goniometer, for the following reasons:

(i) The goniometer motor runs at a speed determined by the line frequency of its electrical supply, whereas the internal timer in an X-ray scaler/timer or the time base of a 
computer controller is likely to be quartz crystal controlled. The line frequency, although highly stable over a long period, may vary by as much as $0.5 \%$ in the short term, with consequent variation in motor speed. This can produce an error in the apparent specimen position (estimated from elapsed time) of up to $25^{\circ}$ in the azimuthal angle, during the course of a typical pole figure measurement. This problem has also been mentioned by Hutchinson. ${ }^{2}$

(ii) When switched on, the goniometer motor takes several seconds to attain constant speed with the result that, in a typical goniometer configuration, an error of perhaps $1 \frac{1}{2^{\circ}}-2^{\circ}$ in specimen azimuth position results from using elapsed time to determine specimen rotation.

One way of eliminating both of these potential sources of error is to trigger the data read-out directly from the specimen rotation. Unfortunately, the built-in angle marker switch is only activated once for each $360^{\circ}$ of azimuth rotation, so cannot be used for this purpose. However, by monitoring the rotation of the goniometer motor, the specimen's position can be determined with precision. The circuit described below accomplishes this by the use of an optical sensor which is activated by reflective strips mounted on the motor armature. The output from the sensor is processed by a presettable counter circuit, producing an output pulse after each user-selectable increment in azimuth rotation. In the most common goniometer configuration, the increment can be varied from $0.1^{\circ}$ to $9.9^{\circ}$ in steps of $0.1^{\circ} .^{*}$ These pulses can then be used to activate the data read-out and scalar reset as required. The unit uses readily available and inexpensive parts, with CMOS integrated circuits. These will accept power supplies from 3-15 volts, and the unit may thus be powered by the DC supply from almost any solid state $x$-ray scaler-timer, no separate power supply being needed.

\section{DESCRIPTION}

The circuit schematic is shown in Figure 1. The following circuit description relates to a goniometer configuration in which the azimuth angle rotates a nominal $360^{\circ}$ in five minutes $\left(60 \mathrm{~Hz}\right.$ line frequency) or $360^{\circ}$ in six minutes ( $50 \mathrm{~Hz}$ line frequency). Under these conditions the motor makes 25 Revolutions for each degree of specimen rotation. A $15 \mathrm{~mm}$ wide band at the bottom of the motor armature is painted matt-black, and two $10 \mathrm{~mm}$ wide strips of aluminum foil are cemented onto this band, at diametrically opposite points on the armature. The optical sensor is mounted on an aluminum bracket screwed to the gear box as shown in Figure 2 , so that it is "looking at" the black band. When the goniometer is running, the sensor sees a reflective strip pass

*The specimen rotation is not, of course, continuous but in steps of $0.5^{\circ}$ or $1^{\circ}$ according to the setting of the goniometer. Thus, if an increment of $6.6^{\circ}$ were specified for the output pulses from the unit, there would be ten pulses output in the time taken for the specimen to rotate $66^{\circ}$. 


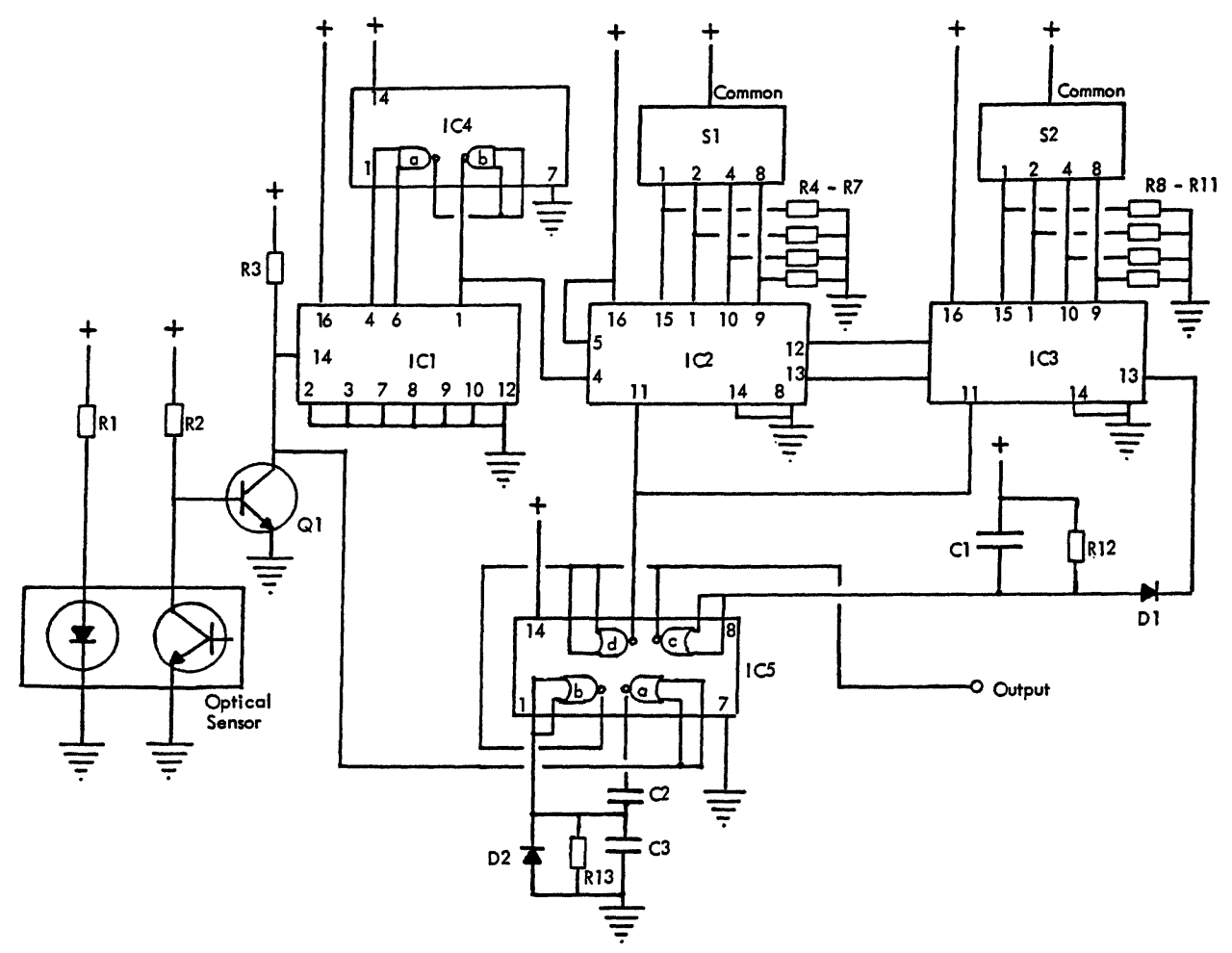

Figure 1. Schematic circuit of the unit.

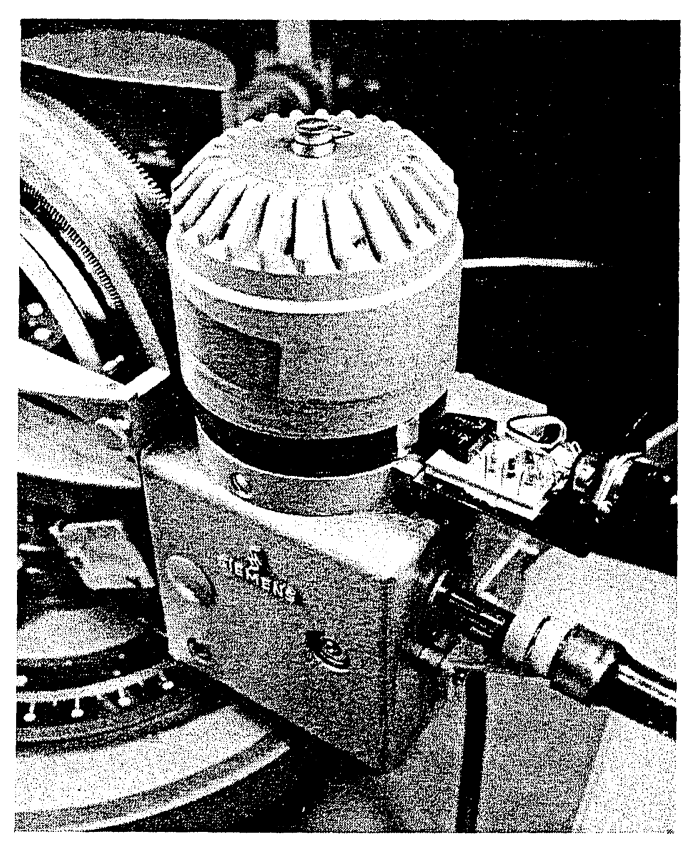

Figure 2. Showing the mounting of the sensor assembly on the texture goniometer. 
fifty times for every degree of specimen rotation.

The optical sensor is a TIL 139 which contains an infrared emitting diode and a photo transistor in a single package. When a reflecting strip on the goniometer motor armature passes the sensor, the photo transistor conducts, causing its collector to go negative. RI is to limit the current through the I.R. emitting diode to about $20 \mathrm{~mA}$, and is given by $\mathrm{R}=$ $50(\mathrm{~V}-2)$ ohms where $\mathrm{V}$ is the power supply voltage (see below). Ql amplifies and inverts the voltage pulse, which is then applied to the clock input of ICl, which is a CMOS 4018 presettable divide by $\mathrm{N}$ counter, set up to divide by 5 [by gates IC4 (a) and IC4(b)]. The output of ICI (from pin 1) now consists of 10 pulses for every degree of specimen rotation. This pulse train is fed into the "count down" input of IC2, which is cascaded with IC3 to form a presettable, two-decade counter. The counter is preset by the BCD thumb wheel switches SI and S2. Whenever the preset count is reached, an output pulse is generated at pin 13 of IC 3 .

From the foregoing, it will be realized that if $\mathrm{s} 2$ and $S I$ are set to $P$ and $Q$, respectively, an output pulse results for every $P+(Q / 10)$ degrees of specimen rotation. The polarity of this pulse is negative.

The output pulse is stretched to a duration of $\sim 1 \mathrm{~ms}$ by the network DI, Cl, RI2 and inverted through IC5(c). The pulse is also fed to IC5(d), whose output resets the counters IC2 and IC 3 .

IC5 (a) and (b) with associated components cause the counters to be reset whenever the stream of incoming pulses stops, ready for the start of the next run.

The output, thus, consists of a stream of positive pulses determined by the goniometer position. If negative pulses are required, one of the unused gates of IC4 may be used to invert and buffer the output.

\section{POWER REQUIREMENTS}

The circuit will work satisfactorily with a power supply voltage from 5-15 volts DC, although RI must be adjusted to suit the supply voltage (see above). The current consumption is $\sim 20 \mathrm{~mA}$, practically all of which is taken by the I.R. emitting diode in the optical sensor. All modern $X-r a y$ scalers have a power supply capable of meeting these requirements.

\section{INTERFACING WITH X-RAY SCALER}

The output drive capability of this unit is that of a cMos logic gate. This will interface directly with a scaler using CMOS, PMOS, NMOS or low power TTL logic inputs, and with most forms of discrete component inputs (such as the G.E. 700 series). If the scaler input is standard TTL it will be necessary to add a buffer, to increase the current sinking ability of the output. (A 74C902 CMOS-TTL buffer, or even a single NPN transistor can be used for this purpose. 


\section{OTHER GONIOMETER CONFIGURATIONS}

The unit described above is set up so that switches s2 and $\mathrm{Sl}$ determine the specimen rotation in degrees and tenths of degrees for a goniometer configuration in which one degree of rotation is accomplished by 25 revolutions of the motor. The other common configuration requires 50 revolutions to obtain 1 degree of rotation. This may be easily accommodated by placing only one reflecting strip on the motor armature.

\section{CONSTRUCTION}

In the prototype unit, the optical sensor and components R1, R2, R3 and Q1 were mounted on a small printed circuit board screwed to the goniometer gear box. The remainder of the circuit was assembled on a small perforated laminate board, Figure 3, and mounted in the electronics console of the X-ray unit used (G.E. 700 Series). As all the signals to be processed are low frequency, the layout of components is not critical. The specifications of the components are given in Table $I$.

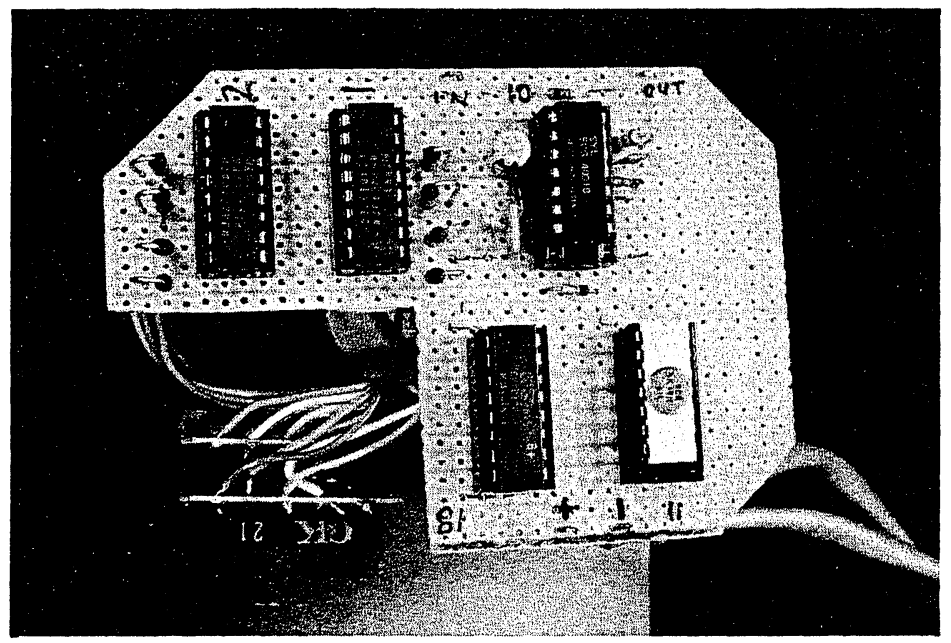

Figure 3. The prototype unit.

\section{ACKNOWLEDGEMENTS}

The author would like to thank Drs. G. J. Davies, D. J. Goodwill and P. P. Morris for discussions, and Mr. K. Page for drawing his attention to the TIL 139 sensor. The work was performed under the support of the National Science Foundation contract DMR 78-18586. 
TABLE I

The Specifications of the Components

$\begin{array}{ll}\text { Optical Sensor } & \text { TIL } 139 \text { (Texas Instruments) } \\ \text { Q1 } & \text { 2N3904 (or any general purpose NPN } \\ \text { transistor) } & 4018 \text { presettable divide by N counter } \\ \text { IC1 } & 40192 \text { presettable up/down decade counter } \\ \text { IC2, IC3 } & 4011 \text { quad } 2 \text { input NAND gate } \\ \text { IC4 } & 4001 \text { quad } 2 \text { input NOR gate } \\ \text { IC5 } & \text { 1N4148 } \\ \text { D1, D2 } & 0.01 \text { microfarad } \\ \text { C1 } & 0.47 \text { microfarad } \\ \text { C2 } & 0.1 \text { microfarad } \\ \text { C3 } & \text { See text } \\ \text { R1 } & 150 \text { kohm } \\ \text { R2 } & 22 \text { kohm } \\ \text { R3 } & 100 \text { kohm } \\ \text { R4-R12 } & 680 \text { kohm } \\ \text { R13 } & \text { Binary coded decimal (BCD) thumb wheel } \\ \text { S1, S2 } & \text { switch }\end{array}$

\section{REFERENCES}

1. Siemens Pole Figure Goniometer, Operating Instructions, Bereich Mess and Prozess technik, Karlsruhe, FDR (1972).

2. M. Hatherly and W. B. Hutchinson, An Introduction to Textures in Metals, The Institution of Metallurgists, London, 1979. 